\title{
Compact and Wide Stopband Lowpass Filter Using Open Complementary Split Ring Resonator and Defected Ground Structure
}

\author{
S. S. KARTHIKEYAN ${ }^{1}$, Rakhesh Singh KSHETRIMAYUM ${ }^{2}$ \\ ${ }^{1}$ Dept. of Electronics Engineering, Indian Inst. of Information Technology, Design and Manufacturing, \\ Kancheepuram Chennai, 600127, India \\ ${ }^{2}$ Dept. of Electronics and Electrical Engineering, Indian Inst. of Technology Guwahati, Guwahati, Assam, 781039, India \\ ssk@iiitdm.ac.in, krs@iitg.ac.in
}

\begin{abstract}
A compact $\left(0.16 \lambda_{g} \times 0.08 \lambda_{g}\right)$ and wide stopband lowpass filter design using open complementary split ring resonator (OCSRR) and defected ground structure (DGS) is presented in this paper. Low pass filter is constructed using two cascaded stages of OCSRR. Since the rejection bandwidth of the OCSRR is narrow, tapered dumbbell shaped DGS section is placed under the OCSRR to enhance the bandwidth. The cutoff frequency $\left(f_{c}\right)$ of the proposed lowpass filter is $1.09 \mathrm{GHz}$. The rejection bandwidth of the filter covers the entire ultra wideband spectrum. Hence the spurious passband suppression is achieved up to $10 f_{c}$. The designed filter has been fabricated and validated by experimental results.
\end{abstract}

\section{Keywords}

Lowpass filter, defected ground structure (DGS), open complementary split ring resonator (OCSRR)

\section{Introduction}

On the motive of conventional filter design methods such as stepped impedance and open stub suffering from poor skirt characteristics and spurious passband, researchers have paid great attention over the past few years in designing a compact and sharp cutoff lowpass filters with spurious free wide stopband. Lowpass filter using different defected ground structures (DGS), photonic bandgap (PBG) structures and electromagnetic bandgap structures have been proposed in various literatures [1-9]. Sharp cutoff and low insertion loss lowpass filter using a complementary split ring resonator (CSRR) is reported in [3]. Cascaded stages of CSRR with open stub configuration, exhibiting sharp cutoff lowpass response are presented in [4]. Planar photonic bandgap (PBG) structures involving compact microstrip resonating cell (CMCR) are utilized to design a lowpass filter with wide stopband [5]. Recently, a lowpass filter with very sharp transition band using OCSRR is demonstrated in [6]. In this paper, a new technique is proposed to reduce the size of the lowpass filter and to obtain the wide spurious free stopband. The lowpass filter is designed using open complementary split ring resonator and a tapered dumbbell shaped DGS is placed under the OCSRRs to extend the stopband. The proposed lowpass filter is smaller and outperforming the existing filters in terms of insertion loss level, roll off rate and rejection bandwidth. Figure 1 shows the schematic of OCSRR printed on the microstrip line. This structure is first proposed in [6] for designing a sharp cutoff lowpass filter with compact size. OCSRR is a negative image of the open split ring resonator. To study the frequency response characteristics of the OCSRR, this structure is designed on Rogers 5880 substrate with a dielectric constant of 2.2 and a substrate thickness of $0.381 \mathrm{~mm}$ and simulated using an Ansys full wave simulator.

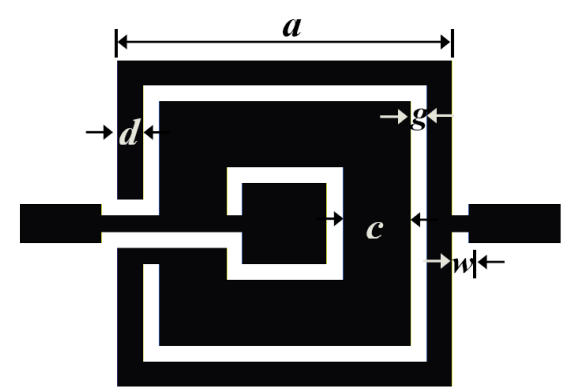

Fig. 1. Layout of the OCSRR.

The dimensions of the OCSRR are as follows: $a=11 \mathrm{~mm}, \quad c=3.4 \mathrm{~mm}, \quad d=0.5 \mathrm{~mm}, \quad g=0.5 \mathrm{~mm}$ and $w=0.5 \mathrm{~mm}$. At the resonant frequency, the resonator is left open and the transmitted energy is reflected back to the source causing a transmission zero. The simulated scattering parameters of the OCSRR are depicted in Fig. 2.

A transmission zero is observed at the resonance frequency $f_{0}=2.46 \mathrm{GHz}$. Since the OCSRR is printed on the microstrip itself, it will change the line inductance and capacitance resulting in a slow wave effect. The slow wave factor $S W F$ of the line loaded with the OCSRR is calculated by the method described in [7] using (1) and plotted in Fig. 3. 


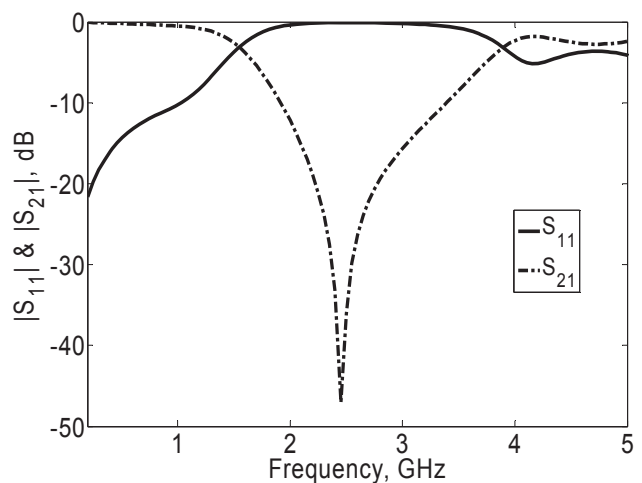

Fig. 2. Simulated scattering parameters.

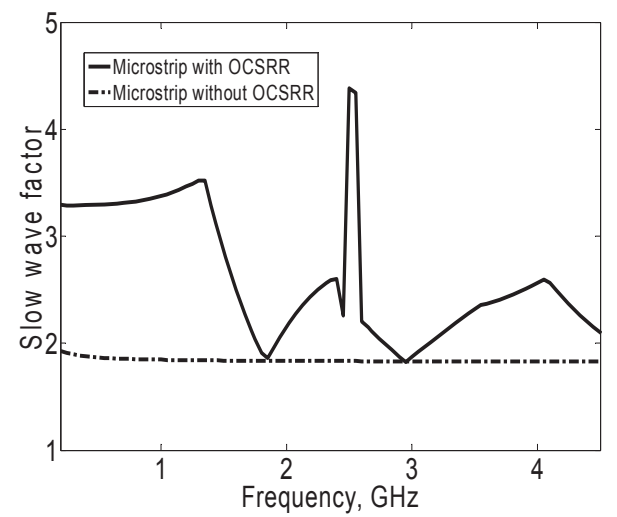

Fig. 3. Slow wave factor of the microstrip line loaded with OCSRR.

$$
S W F=\frac{\lambda_{0} \Delta_{\theta}}{360 L}+\sqrt{\varepsilon_{e f f}}
$$

where $L$ is the physical length of the line, $\Delta_{\theta}$ is the phase difference between the microstrip line with OCSRR and without OCSRR. From the plot, it is observed that the SWF of the microstrip line loaded with OCSRR is increased by $73 \%$ compared to the microstrip line without OCSRR. Further $S W F$ is increased up to $130 \%$ near the resonant frequency. So the increase in $S W F$ validates the size reduction capability of the OCSRR for microwave applications.

\section{Modeling of OCSRR}

During the resonant condition of the OCSRR, the transmitted energy is reflected back to the source causing a transmission zero. The surface current distribution of the OCSRR at the resonant frequency is depicted in Fig. 4.

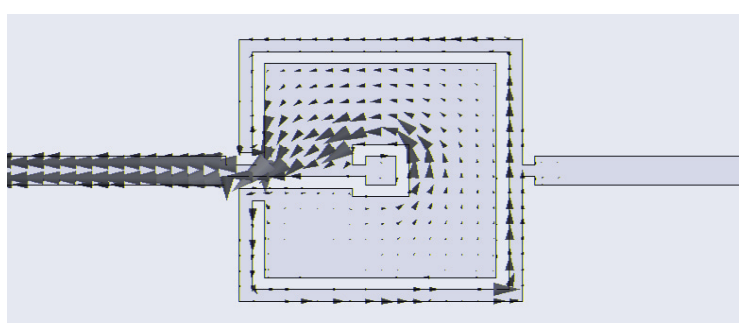

Fig. 4. Current distribution of OCSRR at the resonant frequency.

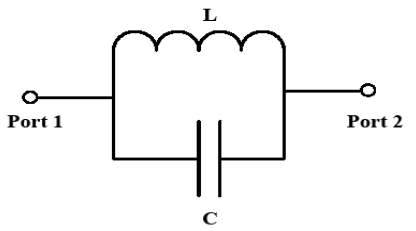

Fig. 5. Lumped element model of OCSRR.

We can model the OCSRR as a parallel resonant circuit as shown in Fig. 5. Here $L$ is the inductance of the rings and $C$ is the capacitance between the rings. The values of equivalent circuit can be extracted using the procedure described in [1] using (2) and (3).

$$
\begin{gathered}
C=\frac{\omega_{c}}{2 Z_{0}\left(\omega_{0}^{2}-\omega_{c}^{2}\right)}, \\
L=\frac{1}{4 \pi^{2} f_{0}^{2} C}
\end{gathered}
$$

where $\omega_{\mathrm{c}}$ and $\omega_{0}$ are the cutoff frequency and attenuation pole frequency respectively. The extracted equivalent circuit values are $C=0.6664 \mathrm{pF}$ and $L=6.2875 \mathrm{nH}$ at the resonant frequency $f_{0}=2.46 \mathrm{GHz}$.

The equivalent circuit is simulated using AWR Microwave office and results are plotted in Fig. 6b. The small discrepancy between EM simulation and circuit simulation may be due to the negligence of losses in the resonant circuit.

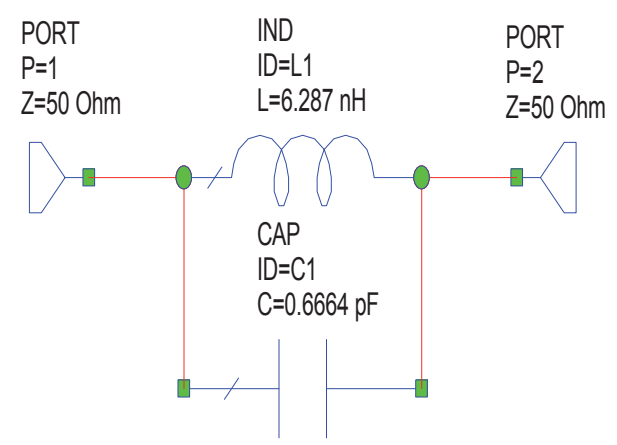

(a) Schematic of the OCSRR equivalent circuit in AWR Microwave office.

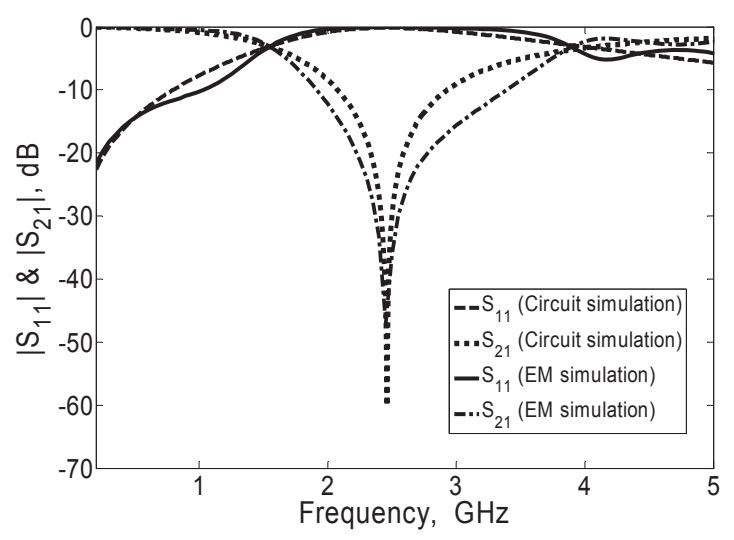

(b) Simulated scattering parameters of OCSRR.

Fig. 6. Schematic and simulated results of OCSRR. 


\section{Lowpass Filter Design using OCSRR}

From the scattering parameters of the OCSRR, it can be observed that the rejection bandwidth of the OCSRR is narrow and not suitable for lowpass filter application.

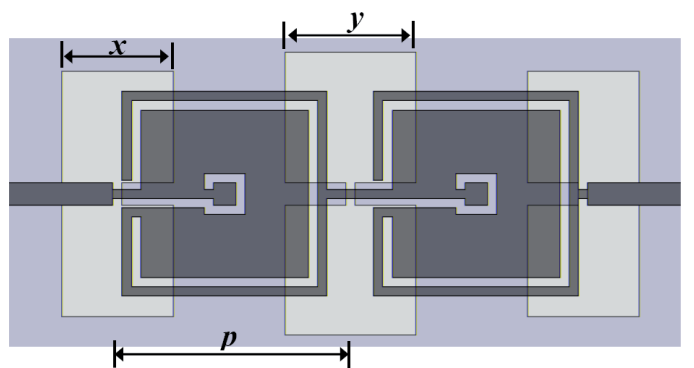

(a)

Schematic of the proposed LPF $(x=5.5 \mathrm{~mm}$, $y=7.2 \mathrm{~mm}, p=12.5 \mathrm{~mm})$

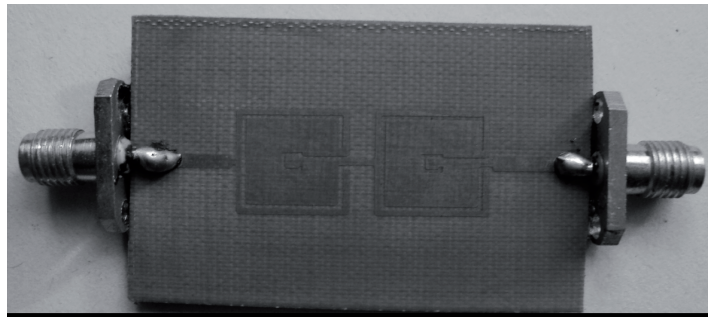

(b) Top view of the fabricated prototype.

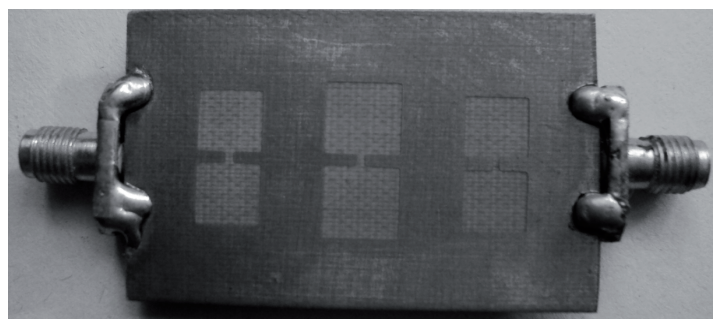

(c) Bottom view of the fabricated prototype.

Fig. 7. Snapshots of the schematic and fabricated filter.

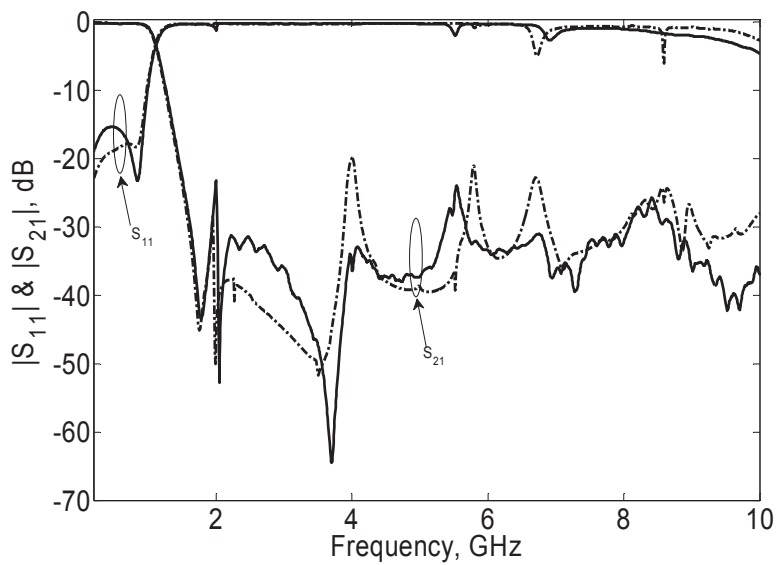

Fig. 8. Scattering parameters of the proposed LPF (Solid line - fabricated, dashed line - simulated).
Therefore, by cascading the number of OCSRRs [6], the required wide stop bandwidth can be achieved. But this technique increases the size of the filter. Hence, in this proposed technique, tapered dumbbell shaped DGSs are placed underneath the OCSRR loaded microstrip line. Since DGSs are etched out from the ground plane, length of the filter is unaltered. Fig. 7 shows the schematic and the fabricated photograph of the proposed LPF, in which two OCSRR cells are cascaded. The dark portion represents the top side and the lighter portion represents the bottom side of the filter. The size of the OCSRR is same as mentioned in the previous section. The size of the DGS is optimized to overlap the stopband of DGS section with the OCSRR stopband to obtain a very wide stopband.

Simulated and measured scattering parameters of the proposed LPF are shown in Fig. 8. The proposed filter has a $3 \mathrm{~dB}$ cutoff frequency of $1.09 \mathrm{GHz}$ and low insertion loss of less than $0.25 \mathrm{~dB}$ in the passband. The insertion loss suddenly drops to $20 \mathrm{~dB}$ at $1.43 \mathrm{GHz}$ which indicates that the transition bandwidth is only $0.34 \mathrm{GHz}$. The return loss in the passband is well above $15 \mathrm{~dB}$. The simulated stopband rejection of the filter is better than $20 \mathrm{~dB}$ up to $10 \mathrm{GHz}$, which is nearly ten times the cutoff frequency. The selectivity of the filter is $48.57 \mathrm{~dB} / \mathrm{GHz}$ calculated using (4)

$$
\xi=\frac{\alpha_{\min }-\alpha_{\max }}{f_{s}-f_{c}}
$$

where $\xi$ is selectivity of the filter, $\alpha_{\min }$ and $\alpha_{\max }$ are the attenuation points at $20 \mathrm{~dB}$ and $3 \mathrm{~dB}$ respectively, and $f_{s}$ is the $20 \mathrm{~dB}$ stopband frequency. Designs are fabricated and are validated using Rohde \& Schwarz Vector Network Analyzer. Manual calibration is done before taking the measurements.

Compared to the work already reported, the proposed filter size is compact and provides a wide stopband rejection. This proposed lowpass filter is much better than the existing highly efficient filters reported in [4] and [6] in terms of stopband rejection width. The proposed lowpass filter has comparable rejection bandwidth with the low pass filter in [5] but it is much more compact than filters reported in [8] and [9] as depicted in Tab. 1.

\begin{tabular}{|l|c|c|c|}
\hline \multicolumn{1}{|c|}{ Structure } & $\begin{array}{c}\text { Cut-off } \\
\text { frequency } f_{\mathrm{c}}\end{array}$ & Size & $\begin{array}{c}\text { Stop band- } \\
\text { width }\end{array}$ \\
\hline CSRR [4] & $1.1 \mathrm{GHz}$ & $0.32 \lambda_{\mathrm{g}} \times 0.18 \lambda_{\mathrm{g}}$ & $5 f_{\mathrm{c}}$ \\
\hline Tapered CMRC [5] & $1.3 \mathrm{GHz}$ & $0.62 \lambda_{\mathrm{g}} \times 0.19 \lambda_{\mathrm{g}}$ & $10 f_{c}$ \\
\hline OCSRR only [6] & $1.33 \mathrm{GHz}$ & $0.81 \lambda_{\mathrm{g}} \times 0.19 \lambda_{\mathrm{g}}$ & $4 f_{c}$ \\
\hline $\begin{array}{l}\text { DGS and radial stub } \\
\text { [8] }\end{array}$ & $2.5 \mathrm{GHz}$ & $0.26 \lambda_{\mathrm{g}} \times 0.167 \lambda_{\mathrm{g}}$ & $14 f_{c}$ \\
\hline $\begin{array}{l}\text { Circular patch and } \\
\text { open stub [9] }\end{array}$ & $1.26 \mathrm{GHz}$ & $0.12 \lambda_{\mathrm{g}} \times 0.29 \lambda_{\mathrm{g}}$ & $12 f_{c}$ \\
\hline Proposed work & $1.09 \mathrm{GHz}$ & $0.16 \lambda_{\mathrm{g}} \times 0.08 \lambda_{\mathrm{g}}$ & $10 f_{c}$ \\
\hline
\end{tabular}

Tab. 1. Comparison of the performance of lowpass filters. 


\section{Conclusion}

This paper presents a new technique to design a lowpass filter with compact dimension and wide rejection bandwidth. By overlapping the stopand of the OCSRR and DGS, wide stopband is achieved. Design technique is well validated with the experimental results. The proposed filter shows a very good performance in terms of low insertion loss, sharp roll off, wide rejection bandwidth and compact design.

\section{References}

[1] AHN, D., PARK, J.-S., KIM, C.-S., KIM, J., QIAN, Y., ITOH, T. A design of the low-pass filter using the novel microstrip defected ground structure. IEEE Transactions on Microwave Theory and Techniques, 2001, vol. 49, no. 1, p. 86-93. DOI: $10.1109 / 22.899965$

[2] ABDEL-RAHMAN, A. B., VERMA, A. K., BOUTEJDAR, A., OMAR, S. Control of bandstop response of Hi-Lo microstrip lowpass filter using slot in ground plane. IEEE Transactions on Microwave Theory and Techniques, 2004, vol. 52, no. 3, p. 1008 to 1013. DOI: $10.1109 /$ TMTT.2004.823587

[3] MANDAL, M., MONDAL, P., SANYAL, S., CHAKRABARTY, A. Low insertion-loss, sharp-rejection and compact microstrip lowpass filters. IEEE Microwave and Wireless Components Letters, 2006, vol. 16, no. 11, p. 600-602. DOI: 10.1109/LMWC.2006.884777

[4] WU, B., LI, B., LIANG, C. Design of lowpass filter using a novel split-ring resonator defected ground structure. Microwave and Optical Technology Letters, 2007, vol. 49, no. 2, p. 288-291. DOI: 10.1002/mop.22111

[5] LI, L., LI, Z.-F., WEI, Q.-F. Compact and selective lowpass filter with very wide stopband using tapered compact microstrip resonant cells. IEEE Electronics Letters, 2009, vol. 45, no. 5, p. 267-268. DOI: 10.1049/el:20092120

[6] AZNAR, F., VELEZ, A., BONACHE, J., MENES, J., MARTIN, F. Compact lowpass filters with very sharp transition bands based on open complementary split ring resonators. IEEE Electronics Letters, 2009, vol. 45, no. 6, p. 316-317. DOI: $10.1049 / \mathrm{el} .2009 .2854$

[7] LI, H.W., LI, Z.F., SUN, X.W., KURACHI, S., CHEN, J., YOSHIMASU, T. Theoretical analysis of dispersion characteristics of mi- crostrip lines with defected ground structure. Journal of Active and Passive Electronic Devices, 2007, vol. 2, no. 4, p. 315-322.

[8] XU, S., MA, K., MENG, F., YEO, K.S. DGS embedded transformed radial stub for ultra-wide stopband lowpass filter. IEEE Electronics Letters, 2012, vol. 48, no. 23, p. 1473-1475. DOI: $10.1049 / \mathrm{el} .2012 .2568$

[9] KARIMI, G., LALBAKHSH, A., SIAHKAMARI, H. Design of sharp roll-off lowpass filter with ultra wide stopband. Microwave and Wireless Components Letters, 2013, vol. 23, no. 6, p. 303 to 305. DOI: 10.1109/LMWC.2013.2261057

\section{About the Authors ...}

S. S. KARTHIKEYAN was born in Mayiladuthurai, Tamilnadu India. He received the B.E. degree in Electronics and Communication Engineering from Bharathidasan University, Trichy, in 2001 and M.E. in Applied Electronics from Sathyabama University, Chennai, in 2005. He obtained his Doctoral Degree from the Indian Institute of Technology Guwahati, Assam, India in 2011. Currently he is working as an Assistant Professor at the Indian Institute of Information Technology, Design and Manufacturing Kancheepuram, Chennai, India. His research interests include electromagnetic bandgap substrates, microwave filters, metamaterials, microwave sensors, etc.

Rakhesh Singh KSHETRIMAYUM received the B. Tech. (first class honors) degree in Electrical Engineering from the Indian Institute of Technology Bombay, India, in August 2000 and the Ph.D. degree in Electrical and Electronic Engineering from the Nanyang Technological University, Singapore, in June 2005. From 2001 to 2002, he was a Software Engineer at the Mphasis Architecting Value, Pune, India. From 2004 to 2005, he was a Research Associate at the Dept. of Electrical Communication Engineering, Indian Institute of Science Bangalore, India. In May-July 2005, he was a Postdoctoral Scholar at the Dept. of Electrical Engineering, Pennsylvania State University, USA. Since September 2005, he is a faculty member at the Dept. of Electronics and Communication, Indian Institute of Technology Guwahati, India, and presently he is an Associate Professor. His present research interests include microwave communication, UWB and printed antennas. 